\title{
Quaternion Note on the Theory of Confocals.
}

\author{
By Dr C. G. Knotr.
}

(Read and Received 8th March 1918.)

The object of this note is to draw attention to a simple extension of a well-known theorem concerning corresponding points on confocal ellipsoids-an extension which seems to have escaped notice. It has been fumiliar to me for years as an illustration of the quaternion treatment.

As shown long ago by Hamilton and Tait, a system of confocal quadrics may be represented by the equation

$$
S \rho(\phi+h)^{-1} \rho=-1
$$

where $\phi$ is a given self-conjugate linear vector function, $h$ a variable parameter, and $\rho$ the vector to any point on the surface.

The properties of self-conjugate linear vector functions are assumed.

A transformation first given by Tait is

$$
\begin{aligned}
S \rho(\phi+h)^{-1} \rho & =S \rho(\phi+h)^{-\frac{1}{2}}(\phi+h)_{-\frac{1}{2}} \rho \\
& =S(\phi+h)^{-\frac{1}{2}} \rho(\phi+h)^{-\frac{1}{2}} \rho \\
& =\left[(\phi+h)^{-\frac{1}{2}} \rho\right]^{2} .
\end{aligned}
$$

Hence, in the present case, $(\phi+h)^{-\frac{1}{2}} \rho$ is a unit vector $=\alpha$ say; and $\rho=(\phi+h)^{\frac{t}{2}} \alpha$.

Every vector drawn to an ellipsoidal surface may therefore be regarded as derived from a unit vector (a radius vector of the unit sphere) by application of the linear vector function or strain $(\phi+h)^{\frac{1}{2}}$.

Thus, if $\alpha$ and $\beta$ are any vectors of the unit sphere,

$$
\phi^{\frac{1}{2}} \alpha \text { and } \phi^{\dagger} \beta
$$

will be vectors of the ellipsoid represented by $S \rho \phi^{-1} \rho=-1$. 
Similarly,

$$
(\phi+h)^{\frac{1}{2}} \alpha \text { and }(\phi+h)^{\frac{1}{2}} \beta
$$

are the corresponding pair of vectors of the confocal ellipsoid represented by $S_{\rho}(\phi+h)^{-1} \rho=-1$. Thus

and

$$
\begin{array}{ll}
\rho_{1}=\phi^{\frac{1}{2}} \alpha, & \sigma_{1}=(\phi+h)^{\frac{1}{2}} \alpha \\
\rho_{2}=\phi^{\frac{1}{3}} \beta, & \sigma_{2}=(\phi+h)^{\frac{1}{3}} \beta
\end{array}
$$

are pairs of corresponding points on the two confocals.

Then

Similarly,

$$
\begin{aligned}
\left(\rho_{1} \pm \sigma_{2}\right)^{2} & =\left\{\phi^{\frac{1}{2}} \alpha \pm(\phi+h)^{\frac{1}{1}} \beta\right\}^{2} \\
& =S \alpha \phi \alpha \pm 2 S \alpha \phi^{\frac{3}{3}}(\phi+h)^{\frac{1}{2}} \beta+S \beta \phi \beta+h \beta^{2} .
\end{aligned}
$$

$$
\begin{aligned}
\left(\rho_{2} \pm \sigma_{1}\right)^{2} & =\left\{\phi^{\frac{1}{2}} \beta \pm(\phi+h)^{\frac{1}{2}} \alpha\right\}^{2} \\
& =S \beta \phi \beta \pm 2 S \beta \phi^{\frac{1}{2}}(\phi+h)^{\frac{1}{2}} \alpha+S \alpha \phi \alpha+h \alpha^{2} .
\end{aligned}
$$

But since $\alpha^{2}=\beta^{2}=-1$ and $\phi^{\frac{1}{3}}(\phi+h)^{\frac{1}{3}}$ is self-conjugate, these are the same expressions.

Hence

$$
T\left(\rho_{1} \pm \sigma_{2}\right)=T\left(\rho_{2} \pm \sigma_{1}\right)
$$

or the tensors (or lengths) of these pairs of vectors are equal.

The negative sign gives Ivory's well-known theorem that the distance between two points, one on each of two confocals, is equal to the distance between their corresponding points.

The positive sign gives a theorem which, though nearly as simple, does not seem to have been noticed hitherto. The combined theorem may be expressed as follows:-

If $O P, O p$ be vectors drawn from the centre to one ellipsoid, and $O Q, O q$ be drawn to their corresponding points on a confocal ellipsoid, and if parallelograms be constructed on $O P$ and $O q$ and on $O p$ and $O Q$, with $O R$ and $O S$ as their respective diagonals from the centre $O$, then these diagonals are equal, and so also are the diagonals $P q$ and $p Q$.

Or otherwise :- Not only is the distance between two points, one on each of two confocals, equal to the distance between their corresponding points, but the middle points of these distances are equidistant from the centre of the confocals.

A well-known theorem which also follows immediately is

$$
\begin{aligned}
{\rho_{1}}^{2}-\sigma_{1}^{2} & =\left(\phi^{\frac{2}{2}} \alpha\right)^{2}-\left((\phi+h)^{\frac{2}{2}} \alpha\right)^{2} \\
& =S \alpha \phi \alpha-\left(S \alpha \phi \alpha+h \alpha^{2}\right) \\
& =h
\end{aligned}
$$


or the squares of the central radii to two corresponding points differ by the parameter by which the squares of the semi-axes differ.

\section{Cartesian Proof.}

If $a b c, a_{1} b_{1} c_{1}$, are the semi-axes of two confocals, so that $a^{2}=a_{1}{ }^{2}+h_{1}, b^{2}=b_{1}{ }^{2}+h, c^{2}=c_{1}{ }^{2}+h$, and if $(x y z)$ is a point on the one, the corresponding point $\left(x_{1} y_{1} z_{1}\right)$ on the other is given by the relations

$$
\frac{x_{1}}{a_{1}}=\frac{x}{a}, \frac{y_{1}}{b_{1}}=\frac{y}{b}, \frac{z_{\mathrm{J}}}{c_{1}}=\frac{z}{c} .
$$

Let $(X Y Z),\left(X_{1} Y_{1} Z_{1}\right)$ be the coordinates of another pair of corresponding points, then

$$
\begin{aligned}
(X & \left.-x_{1}\right)^{2}+\left(Y-y_{1}\right)^{2}+\left(Z-z_{1}\right)^{2} \\
& =\left(\frac{a}{a_{1}} X_{1}-\frac{a_{1}}{a} x\right)^{2}+\text { etc. } \\
& =\frac{a^{2}}{a_{1}^{2}} X_{1}^{2}-2 X_{1} x+\frac{a_{1}^{2}}{a^{2}} x^{2}+\text { etc. } \\
& =\frac{a_{1}^{2}+h}{a_{1}^{2}} X_{1}^{2}-2 X_{1} x+\frac{a^{2}-h}{a^{2}} x^{2}+\text { etc. } \\
& =\left(X_{1}-x\right)^{2}+\ldots+h-h \\
& =\left(X_{1}-x\right)^{2}+\left(Y_{1}-y\right)^{2}+\left(Z_{1}-z\right)^{2},
\end{aligned}
$$

which establishes Ivory's theorem.

But note that, if the negative signs in the first expressions are changed throughout to positive signs, the proof still holds, or

$$
\begin{aligned}
& \left(X+x_{1}\right)^{2}+\left(Y+y_{1}\right)^{2}+\left(Z+z_{1}\right)^{2} \\
& =\left(X_{1}+x\right)^{2}+\left(Y_{1}+y\right)^{2}+\left(Z_{1}+z\right)^{2},
\end{aligned}
$$

establishing the theorem given above.

It is curious that such a simple generalization should have escaped notice. 